\title{
Programmed death-1 blockade enhances the antitumor effects of peptide vaccine-induced peptide-specific cytotoxic $T$ lymphocytes
}

\author{
YU SAWADA $^{1,2}$, TOSHIAKI YOSHIKAWA ${ }^{1}$, MANAMI SHIMOMURA ${ }^{1}$, TATSUAKI IWAMA ${ }^{1}$, \\ ITARU ENDO $^{2}$ and TETSUYA NAKATSURA ${ }^{1}$ \\ ${ }^{1}$ Division of Cancer Immunotherapy, Exploratory Oncology Research and Clinical Trial Center, \\ National Cancer Center, Kashiwa, Chiba 277-8577; ${ }^{2}$ Department of Gastroenterological Surgery, \\ Yokohama City University Graduate School of Medicine, Kanazawa-ku, Yokohama 236-0004, Japan
}

Received September 7, 2014; Accepted October 17, 2014

DOI: $10.3892 /$ ijo.2014.2737

\begin{abstract}
Novel treatment modalities are required urgently in patients with hepatocellular carcinoma (HCC). A vaccine that induces cytotoxic T lymphocytes (CTLs) is an ideal strategy for cancer, and glypican-3 (GPC3) is a potential option for HCC. Blocking the programmed death-1 (PD-1)/PD-L1 pathway is a rational strategy to overcome tumor escape and tolerance toward CTLs. In the present study, we investigated whether anti-PD-1 blocking antibodies ( $\alpha$ PD-1 Ab) enhanced the number of vaccine-induced peptide-specific CTLs in peripheral blood mononuclear cells (PBMCs) following the administration of GPC3 peptide vaccine to both patients and in a mouse model. The inhibitory receptor PD-1 was highly expressed in ex vivo GPC3-specific CTLs isolated from the PBMCs of vaccinated HCC patients. In vitro, interferon- $\gamma$ induced PD-L1 expression in liver cancer cell lines. In addition, PD-1 blockade increased the number of GPC3-specific CTLs, which degranulate against liver cancer cell lines. In vivo experiments using tumor-bearing mouse models showed that the combination therapy of peptide vaccine and $\alpha \mathrm{PD}-1 \mathrm{Ab}$ suppressed tumor growth synergistically. PD-1 blockade increased the number of peptide-specific tumor-infiltrating $\mathrm{T}$ cells (TILs) and decreased the expression of inhibitory receptors on TILs. This study demonstrated that PD-1/PD-L1
\end{abstract}

Correspondence to: Dr Tetsuya Nakatsura, Division of Cancer Immunotherapy, Exploratory Oncology Research and Clinical Trial Center, National Cancer Center, 6-5-1 Kashiwanoha, Kashiwa 2778577, Japan

E-mail: tnakatsu@east.ncc.go.jp

Abbreviations: HCC, hepatocellular carcinoma; CTL, cytotoxic T lymphocyte; GPC3, glypican-3; PD-1, programmed death-1; PBMC, peripheral blood mononuclear cell; HLA, human leukocyte antigen; IFN- $\gamma$, interferon- $\gamma$; MHC, major histocompatibility complex

Key words: programmed death-1, cytotoxic T lymphocyte, peptide vaccine, glypican-3, hepatocellular carcinoma blockade augmented the antitumor effects of a peptide vaccine by increasing the immune response of vaccine-induced CTLs, and provided a foundation for the clinical development of a combination therapy using a GPC3 peptide vaccine and $\alpha$ PD-1 Ab.

\section{Introduction}

Antigen-specific cancer immunotherapy using the induction of tumor-specific reactions without autoimmunity is a potentially attractive option for the treatment of cancer. However, immunotherapy for hepatocellular carcinoma (HCC) is still in the preclinical or early clinical trial phases (I and II) of development $(1,2)$. Glypican-3 (GPC3), a carcinoembryonic antigen, is overexpressed in $72-81 \%$ of HCC cases, and is correlated with poor prognosis; therefore, it is an ideal target for HCC (3-7). Recently, a phase I clinical study of a GPC3derived peptide vaccine reported its safety and efficacy for the treatment of advanced HCC (8). Although vaccine-induced GPC3-peptide-specific cytotoxic T lymphocytes (CTLs) are often tumor reactive in vitro (9) and correlate with overall survival, no complete response was observed when GPC3 peptide vaccination was used as monotherapy in patients with advanced HCC (8).

Programmed death-1 (PD-1) is expressed on activated $\mathrm{T}$ and $\mathrm{B}$ cells, and elicits inhibitory signals (10). Its ligand PD-L1 is member of the B7 family, and interacts with PD-1 (11). Several studies have shown that the PD-1/PD-L1 pathway plays a critical role in compromised tumor immunity $(12,13)$. PD-1 antibody blockade exerts antitumor effects in clinical trials $(14,15)$. High expression levels of PD-1 on T cells, both in tumor-infiltrating lymphocytes (TILs) and peripheral blood mononuclear cells (PBMCs), were correlated with poor prognosis in HCC patients after surgical resection (16). In addition, PD-L1 expression in HCC was correlated with tumor aggressiveness and postoperative recurrence (17).

In animal models, PD-1 blockade exerts synergistic effects with various tumor vaccines to enhance tumor antigenspecific T cell responses and suppress tumors in vivo (18-20). It was reported that melanoma vaccine-induced CTLs become exhausted, which could be reversed by blocking the inhibitory pathways (21). However, a study evaluating the combination 
of a cancer vaccine and an anti-PD-1 blocking antibody $(\alpha \mathrm{PD}-1 \mathrm{Ab})$ for $\mathrm{HCC}$ has not been conducted. Therefore, the aim of this study was to investigate whether $\alpha$ PD-1 Ab would enhance the antitumor effects of a peptide vaccine by analyzing CTLs isolated from the PBMCs of vaccinated patients, as well as from a mouse model.

\section{Materials and methods}

Patient samples. Three clinical trials were conducted using GPC3-derived peptide vaccines. A phase I trial $(n=33)$ was performed in patients with advanced or metastatic HCC (8) (University Hospital Medical Information Network Clinical Trials Registry; UMIN-CTR no. 000001395). Subsequently, a phase II trial was performed using a GPC3-derived peptide vaccine as an adjuvant therapy in patients with HCC (UMIN-CTR: 000002614, on-going). Finally, a pilot study of liver biopsies taken before and after GPC3 peptide vaccination is being performed for advanced HCC (UMIN-CTR: 000005093, on-going). These trials were approved by the Ethics Committee of the National Cancer Center, Japan, and conformed to the ethical guidelines of the 1975 Declaration of Helsinki. All patients were enrolled after providing written informed consent. Patients were injected intradermally with HLA-A24-restricted GPC3 ${ }_{298-306}$ (EYILSLEEL) or HLA-A2restricted GPC $3_{144-152}$ (FVGEFFTDV) peptide vaccines emulsified with incomplete Freund's adjuvant (IFA, Montanide ISA-51VG; SEPPIC).

Peripheral blood $(30 \mathrm{ml})$ was obtained at the National Cancer Center Hospital East. PBMCs were isolated using standard Ficoll density gradient centrifugation from buffy coats. The remaining PBMCs were used after immunological monitoring in clinical trials. The immunological analyses were approved by the Ethics Committee of the National Cancer Center, Japan.

Cell lines. The human liver cancer cell lines SK-Hep-1 (GPC3-, HLA-A*02:01/A*24:02), SK-Hep-1/GPC3 (GPC3 ${ }^{+}$, HLA-A*02:01/A*24:02), and HepG2 (GPC3 ${ }^{+}$, HLA-A*02:01/ $\left.A^{*} 24: 02\right)$ were available in our laboratory and were used as the target cells $(6,9)$. SK-Hep-1/GPC3 is an established stable GPC3-expressing cell line that was transfected with the human GPC3 gene, whereas SK-Hep-1/vec is an established counterpart cell line that was transfected with an empty vector. The mouse lymphoma cell line RMA (OVA-, H-2K ${ }^{\mathrm{b}}$ ) was provided by Dr Yasuharu Nishimura (Kumamoto University, Japan). Cells were cultured at $37^{\circ} \mathrm{C}$ in RPMI-1640 or DMEM (SigmaAldrich) supplemented with $10 \%$ fetal bovine serum (FBS), $100 \mathrm{U} / \mathrm{ml}$ penicillin and $100 \mu \mathrm{g} / \mathrm{ml}$ streptomycin in a humidified atmosphere containing $5 \% \mathrm{CO}_{2}$.

Synthetic peptides and cytokines. The peptides used in this study were as follows: HLA-A*02:01-restricted GPC $3_{144-152}$ (FVGEFFTDV) peptide (American Peptide Co.), HLA-A*24: 02-restricted GPC3 ${ }_{298-306}$ (EYILSLEEL) peptide (American Peptide Co.), HLA-A*02:01-restricted human immunodeficiency virus (HIV) $)_{77-85}$ (SLYNTYATL) peptide (ProImmune), and $\mathrm{H}-2 \mathrm{~K}^{\mathrm{b}}$-restricted ovalbumin (OVA) ${ }_{257-264}$ (SIINFEKL) peptide (AnaSpec). The peptides were dissolved and diluted in $7 \% \mathrm{NaHCO}_{3}$ or dimethyl sulfoxide (DMSO). Where appro- priate, liver cancer cell cultures were treated with $100 \mathrm{U} / \mathrm{ml}$ recombinant interferon (IFN) $-\gamma$ (PeproTech).

Ex vivo Dextramer staining and flow cytometry. PBMCs were stained using HLA-A*02:01 Dextramer-RPE [GPC $3_{144-152}$

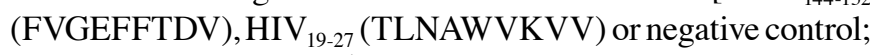
Immudex] and HLA-A*24:02 Dextramer-RPE [GPC3 ${ }_{298-306}$ (EYILSLEEL), HIV 583-591 (RYLKDQQLL); Immudex] for $15 \mathrm{~min}$ at room temperature, followed by anti-CD8-FITC (clone T8, Beckman Coulter), anti-PD-1-APC (clone EH12.2H7, BioLegend), or isotype control-APC (clone MOPC-21, BioLegend) for $20 \mathrm{~min}$ at $4^{\circ} \mathrm{C}$. Flow cytometry was performed using a FACSCanto II (BD Biosciences).

Blocking antibody. GPC3 peptide-specific CTL clones were established from PBMCs as described previously (9). The CTL clones were cultured in AIM-V medium (Life Technologies) supplemented with $10 \%$ human $\mathrm{AB}$ serum in the presence of $10 \mu \mathrm{g} / \mathrm{ml}$ anti-PD-1 (clone $\mathrm{J} 116$, eBioscience) or $10 \mu \mathrm{g} / \mathrm{ml}$ control (clone MOPC-21, BioXcell) monoclonal antibodies for 2 days.

CD107a assay. GPC3 peptide-specific CTL clones were incubated with SK Hep-1/vec pulsed with GPC $3_{144-152}$ or HIV H9-27 $_{10}$ peptide and HepG2 at a $1: 1$ ratio for $3.5 \mathrm{~h}$ at $37^{\circ} \mathrm{C}$. CTL clones were stained with anti-CD107a-APC (clone LAMP-1, BD Bioscience) during the incubation period, followed by antiCD8-FITC (clone LT8, ProImmune) for $20 \mathrm{~min}$ at $4^{\circ} \mathrm{C}$.

Mice. Female C57BL/6 mice (6-8 weeks old) were purchased from Japan Charles River Laboratories (Yokohama, Japan), and were maintained under specific pathogen-free conditions. The Animal Research Committee of the National Cancer Center, Japan, approved all studies. All animal procedures were performed according to the guidelines for the Animal Research Committee of the National Cancer Center, Japan. Ether was used for mouse euthanasia and anesthesia.

In vivo tumor growth inhibition assays. It was reported previously that intratumoral (i.t.) injection of $\mathrm{OVA}_{257-264}$ peptide (SIINFEKL) effectively inhibited OVA-negative tumor growth and survival in a peptide vaccine model using C57BL/6 mice (22). RMA cells $\left(1 \times 10^{5}\right.$ cells $\left./ 100 \mu 1 \mathrm{PBS}\right)$ were implanted on the backs of C57BL/6 mouse on day 0 . They were then injected with 50- $\mu$ g peptide mixed with an equal volume of incomplete Freund's adjuvant (IFA, Montanide ISA-51VG; SEPPIC) on days 7 and 14. The total volume of injected vaccine solution was $100 \mu \mathrm{l}$ in all experiments. For in vivo therapeutic experiments, anti-mouse PD-1 (clone 4H2) and control Ab (clone MOPC-21, BioXcell) were provided by Ono Pharmaceutical Co., Ltd. The anti-mouse PD-1 Ab (clone 4H2) used in the present study is a chimeric rat Ab containing the murine IgG1 Fc region (23). Anti-PD-1 or control Abs (200 $\mu \mathrm{g} /$ day) were injected intraperitoneally (i.p.) on days 7 and 14. Tumor volume was monitored twice per week, and was calculated using the following formula: tumor volume $\left(\mathrm{mm}^{3}\right)=\mathrm{a} \times \mathrm{b} \times \mathrm{b} \times 0.5$, where $\mathrm{a}$ is the longest diameter, $\mathrm{b}$ is the shortest diameter, and 0.5 is a constant to calculate the volume of an ellipsoid. Mouse health, behavior and mortality were checked daily. All mice were maintained until they showed signs of morbidity or the 
A

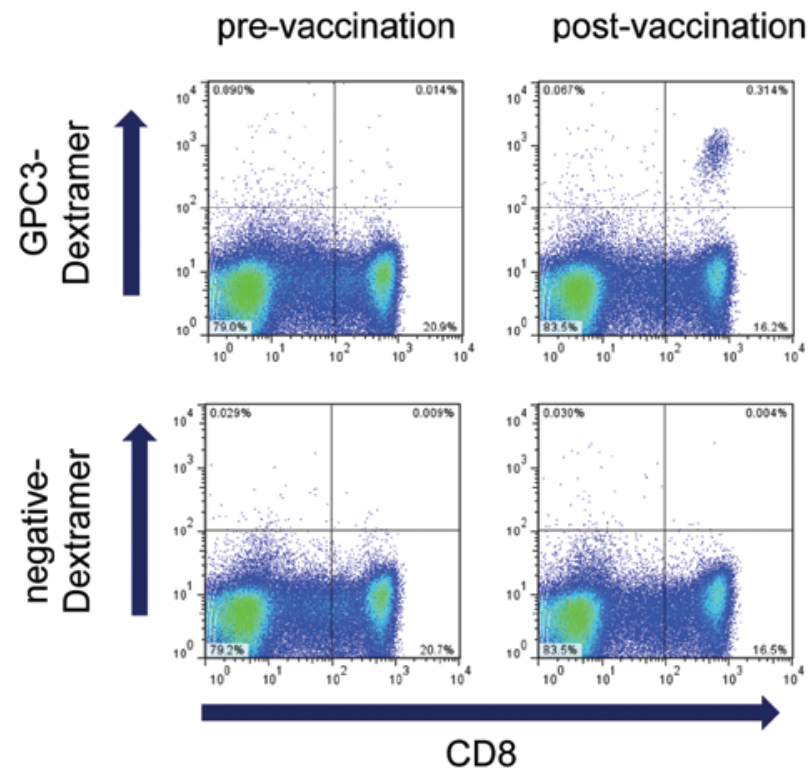

B

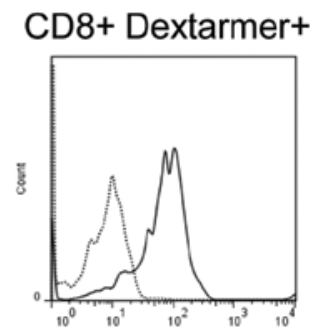

CD8+ Dextarmer-

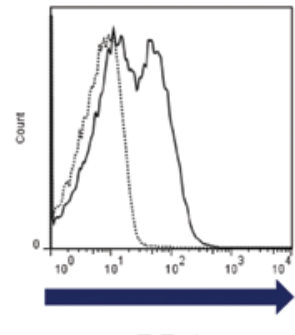

PD1
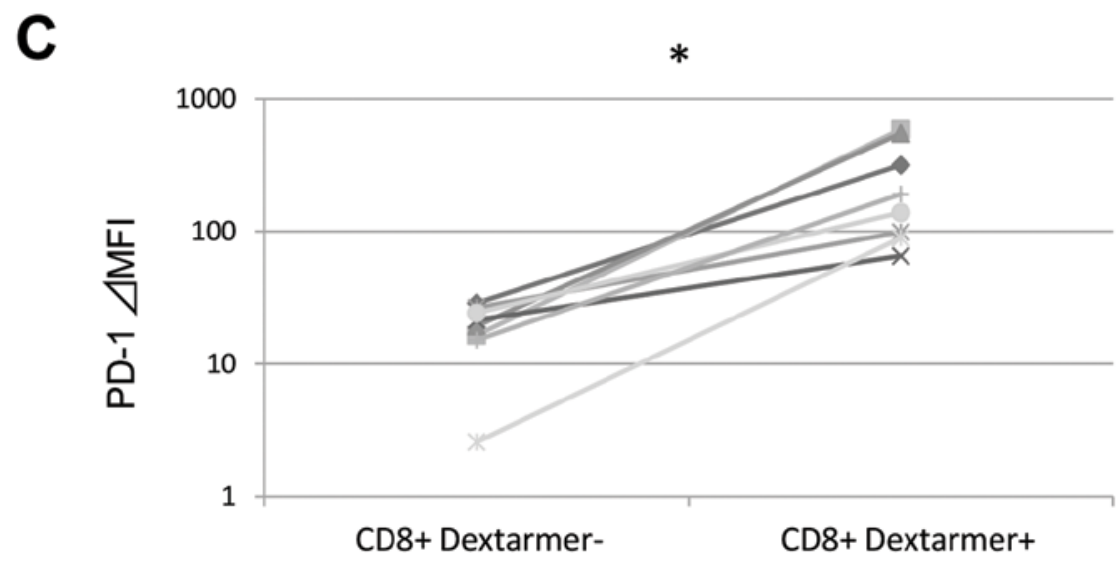

Figure 1. PD-1 expression on GPC3-specific CTLs after vaccination. (A) Ex vivo GPC3 Dextramer staining before and after vaccination in a representative case. The frequency of GPC3 peptide-specific CTLs is indicated as the percentage of the Dextramer-positive CTLs among PBMCs. (B) GPC3-specific CTLs were acquired by gating the CD8-positive/GPC3 Dextramer-positive population. The CD8-positive/GPC3 Dextramer-negative population was used as the control. (C) PD-1 expression on GPC3-specific CD8-positive/Dextramer-positive or -negative populations from eight patient specimens. $\triangle$, MFI, MFI using anti-PD-1 subtracted by that using isotype control. "P<0.05, $\mathrm{n}=8$ using Wilcoxon's signed-rank test.

length or width of the tumors exceeded $30 \mathrm{~mm}$, at which point they were sacrificed for reasons of animal welfare (22).

IFN- $\gamma$ enzyme-linked immunospot (ELISPOT) analysis. The $\mathrm{BD}^{\mathrm{TM}}$ ELISPOT set (BD Biosciences) was used to assess the levels of IFN- $\gamma$, as described previously (24). Briefly, CD8-positive splenocytes $\left(5 \times 10^{5}\right)$ were added to the plate as effector cells. Then, either bone marrow-derived dendritic cells (BM-DCs; $\left.1 \times 10^{5}\right)$ pulsed with OVA peptide $(10 \mu \mathrm{g} / \mathrm{ml}$; as target cells) or non-pulsed BM-DCs ( $1 \times 10^{5}$; as control cells) were added. The plate was then incubated for $37^{\circ} \mathrm{C}$, for $20 \mathrm{~h}$ in the presence of $5 \% \mathrm{CO}_{2}$. Spots were counted automatically using the Eliphoto system (Minerva Tech).

Isolation of mouse tumors and flow cytometry. The mice were sacrificed and the dorsal tumors were dissected, cut into small pieces, and digested with collagenase $(1 \mathrm{mg} / \mathrm{ml})$ for $20 \mathrm{~min}$ at $37^{\circ} \mathrm{C}$. After the intratumoral injection of $\mathrm{OVA}_{257-264}$ peptide, tumor cells were isolated and stained with anti-mouse $\mathrm{H}-2 \mathrm{~K}^{\mathrm{b}}$ bound to $\mathrm{OVA}_{257-264}$ peptide (SIINFEKL)-PE (clone 25-D1.16, BioLegend) or isotype control-PE (MOPC-21, BioLegend). To analyze the local accumulation of antigen-specific CTLs in mice, isolated tumor cells including tumor-infiltrating lymphocytes were stained with $\mathrm{H}-2 \mathrm{~K}^{\mathrm{b}}$ OVA Tetramer-PE [OVA $257-264$ (SIINFEKL); MBL] for $30 \mathrm{~min}$ at room temperature. They were then incubated with anti-mouse CD8-FITC (clone KT15, MBL), anti-mouse PD-1-PE-Cy7 (clone 29F.1A12, BioLegend), anti-mouse CTLA-4-APC (clone UC10-4B, BioLegend), or anti-mouse LAG-3-PerCP-Cy5.5 (clone RTK2071, BioLegend) for $20 \mathrm{~min}$ at $4^{\circ} \mathrm{C}$.

Quantitative real-time PCR. The tumors implanted into mice were dissected. Total RNA was isolated from homogenized tumors using RNeasy mini kit (Qiagen) according to 
Black : IFN- $\mathrm{Y}(+)$

Gray : IFN- $-(-)$

Dot line : isotype control
SK-Hep1/vec

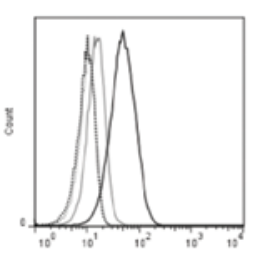

SK-Hep1/GPC3

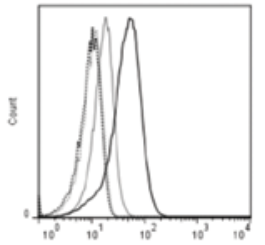

PD-L1
Figure 2. PD-L1 expression in liver cancer cell lines. Liver cancer cell lines were cultured with $100 \mathrm{U} / \mathrm{ml} \mathrm{IFN}-\gamma$ for $24 \mathrm{~h}$. PD-L1 expression was then analyzed using flow cytometry. Two independent experiments were performed, which yielded similar results.

the manufacturer's instructions. First-strand complementary deoxyribonucleic acid (cDNA) was synthesized using a PrimeScript ${ }^{\circledR}$ II first-strand cDNA Synthesis kit (Takara). Quantitative real-time PCR was then performed on an Applied Biosystems 7500 FAST Real-time PCR system using Power SYBR $^{\circledR}$ Green (Applied Biosystems). We assessed the expression of the chemokines CXCL10, CXCL12, and CCL3, and compared them to $\beta$-actin. Data ware analyzed using delta-delta CT methods. Primer sequences of the chemokines were as described (25), and were purchased from Sigma Genosys.

Statistical analysis. All statistical analyses were performed using PASW Statistics software, version 18.0 (SPSS Inc.).
Statistical significance was defined as a value of $\mathrm{P}<0.05$ based on a two-tailed test.

\section{Results}

$P D-1$ expression ex vivo in GPC3 peptide-specific CTLs after vaccination in patients. To investigate whether vaccineinduced CTLs were affected by the PD-1/PD-L1 pathway, we measured the ex vivo expression of PD-1 on vaccine-induced GPC3-specific CTLs using flow cytometry with the GPC3Dextramer. We used PBMCs obtained from eight patients during clinical trials of the GPC3 peptide vaccine. After vaccination, the frequency of GPC3-specific CTLs increased and could be detected ex vivo, as shown in the representative case 1 (Fig. 1A). GPC3-Dextramer-positive CD8 lymphocytes had a higher expression of PD-1 compared with GPC3-Dextramernegative CD8 lymphocytes (Fig. 1B; representative case 1). In all eight patients with detectable GPC3-specific CTLs ex vivo after vaccination, $\mathrm{PD}-1$ expression levels were significantly higher in GPC3-Dextramer-positive CD8 lymphocytes compared with GPC3-Dextramer-negative CD8 lymphocytes (Fig. 1C). Before vaccination, no GPC3-Dextramer-positive CD8 lymphocytes were detected ex vivo; therefore, PD-1 expression was not analyzed.

PD-1 blockade augments the GPC3-specific CTL clones that degranulate against liver cancer cell lines. SK-Hep1/vec, SK-Hep1/GPC3, and HepG2 liver cancer cell lines cultured with IFN- $\gamma$ exhibited marked induction of PD-L1 on their surface (Fig. 2). This suggests that liver cancer cells are invaded by IFN- $\gamma$-producing CTLs via the PD-L1-mediated ligation of PD-1. Previously, several GPC3 peptide-specific CTL clones were established from PBMCs isolated from vaccinated patients. These clones exhibited cytotoxic activity

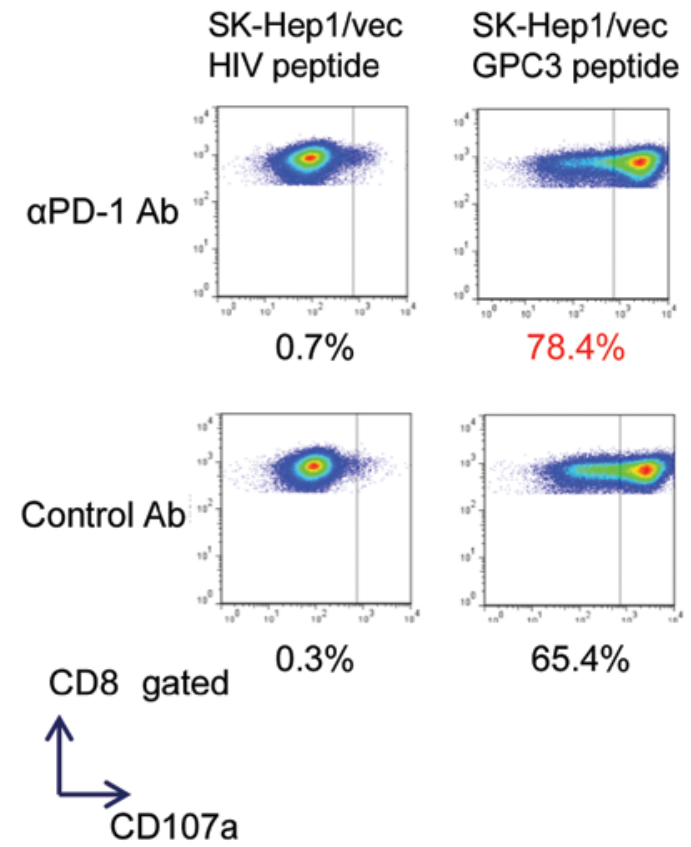

\section{SK-Hep1/vec SK-Hep1/GPC3}

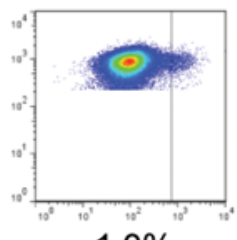

$1.0 \%$

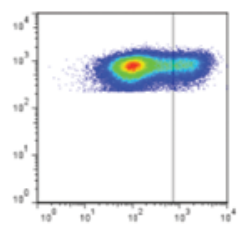

$14.5 \%$

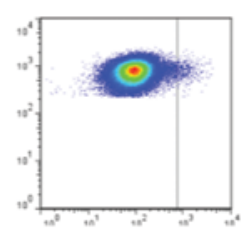

$0.4 \%$

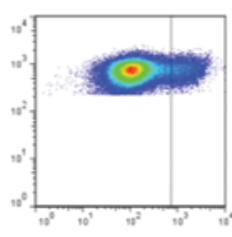

$7.7 \%$
HepG2

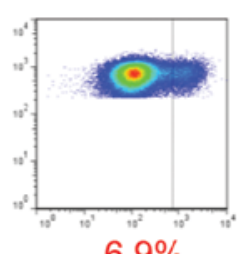

$6.9 \%$

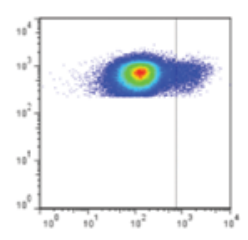

$2.6 \%$

Figure 3. Blocking PD-1 increases GPC3-specific CTL clones that degranulate against liver cancer cell lines. The ratio of GPC3-specific CTL clones that externalized CD107a is shown below each column. The liver cancer cell lines used as the target cell are shown above each column. SK-Hep1/vec (GPC3) cells pulsed with peptide $(1 \mu \mathrm{g} / \mathrm{ml})$ were used as the target cells. The culture conditions are shown in rows. GPC3-specific CTL clones were acquired by gating the CD8-positive population. Two independent experiments were performed, which yielded similar results. 
A

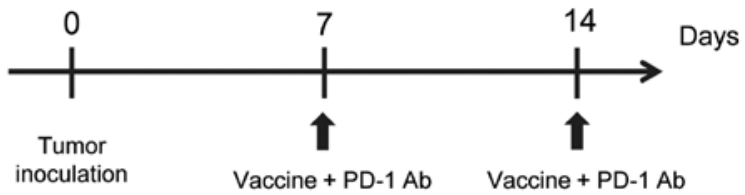

B

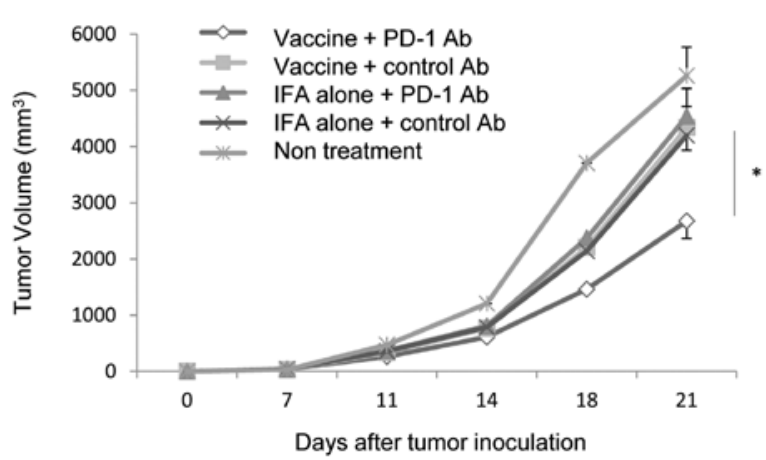

Figure 4. Peptide vaccine and $\alpha$ PD-1 Ab suppress tumor growth synergistically in vivo. (A) Mice implanted with RMA were treated with OVA peptide vaccine or IFA alone in combination with $\alpha$ PD-1 Ab or control Ab on days 7 and 14. (B) Tumor growth was expressed as mean tumor volume; bars, SE. Vaccine, OVA peptide emulsified with IFA; IFA alone, vehicle emulsified with IFA. ${ }^{*} \mathrm{P}<0.05, \mathrm{n}=10$ using Tukey's test. Two independent experiments were performed, which yielded similar results.

against cancer cells expressing GPC3 endogenously $(9,26)$. Therefore, the CD107a (lysosomal-associated membrane protein-1)-mediated externalization of GPC3 peptide-specific CTL clones was examined upon exposure to liver cancer cell lines. The externalization of CD107a could be a surrogate marker to identify the antigen-specific CTLs that degranulate against tumor cells (27). CTL clones mobilized CD107a in response to SK-Hep1/vec pulsed with $\mathrm{GPC} 3_{144-152}$ peptide, SK-Hep-1/GPC3, and HepG2 $\left(\mathrm{GPC}^{+}\right.$, HLA-A $\left.{ }^{*} 02: 01^{+}\right)$, but not in response to pulsed SK-Hep1/vec with $\mathrm{HIV}_{19-27}$ (Fig. 3). Furthermore, PD-1 blockade enriched the population of GPC3-specific CTLs that degranulated against only GPC3-positive liver cancer cell lines (SK-Hep1/vec pulsed with GPC $3_{144-152}$ peptide, SK-Hep1/GPC3 and HepG2). These results suggest that blocking the interaction between PD-1 and PD-L1 enhanced the antitumor effect of CTLs in liver tumor cells that evade CTLs via PD-L1 expression.

Combination of a peptide vaccine and $\alpha P D-1$ Ab suppresses tumor growth in vivo synergistically. Intratumoral injection with OVA $_{257-264}$ peptide (SIINFEKL) effectively inhibited the growth of OVA-negative tumors in a mouse model treated with a peptide vaccine (22). Therefore, we performed in vivo therapeutic experiments using intratumoral OVA peptide vaccine and $\alpha \mathrm{PD}-1 \mathrm{Ab}$ in tumor implanted mice. Mice were implanted with RMA tumor cells on day 0, and established tumors (3-6 $\mathrm{mm}$ in diameter) were treated with OVA peptide emulsified with IFA (vaccine) or vehicle emulsified with IFA (IFA alone) in combination with $\alpha \mathrm{PD}-1 \mathrm{Ab}$ or control $\mathrm{Ab}$ on day 7. An additional dose of vaccine and $\alpha \mathrm{PD}-1 \mathrm{Ab}$ was administered on day 14 after tumor inoculation (Fig. 4A). On day 21, one mouse in the untreated group was dead, and all other mice were alive. The tumor volume of mice treated using the combi- nation therapy of vaccine and $\alpha \mathrm{PD}-1 \mathrm{Ab}$ was significantly less than those treated with the appropriate control (Fig. 4B, n=10). Treatment with vaccine/control Ab or IFA alone/ $\alpha$ PD-1 Ab did not inhibit tumor growth compared with IFA alone/control $\mathrm{Ab}$ treatment. These data suggest that the combination of peptide vaccine and $\alpha \mathrm{PD}-1 \mathrm{Ab}$ had a synergistic antitumor effect.

Vaccine and $\alpha P D-1$ Ab treatment increases the number of peptide-specific CTLs within mouse tumors. The loading of injected peptide onto major histocompatibility complex (MHC) class I molecules in tumor cells in vivo was reported previously using IFN- $\gamma$ ELISPOT assays (22). In the present study, RMA (OVA-, H-2K ${ }^{b}$ ) tumor cells were inoculated onto the backs of C57/BL6 mice. When the tumor diameter reached 3-6 mm, $50 \mu \mathrm{g} \mathrm{H}-2 \mathrm{~K}^{\mathrm{b}}$-restricted $\mathrm{OVA}_{257-264}$ peptide was injected into the tumor. After $96 \mathrm{~h}$, the tumors were dissected, cut into small pieces, and digested using collagenase. To investigate whether the injected peptide was loaded onto the MHC class I molecules in the tumor cells in a solid mass, flow cytometry using anti-mouse $\mathrm{H}-2 \mathrm{~K}^{\mathrm{b}}$ bound to $\mathrm{OVA}_{257-264}$ peptide was performed. The loading of $\mathrm{H}_{-2} \mathrm{~K}^{\mathrm{b}}$-restricted $\mathrm{OVA}_{257-264}$ peptide onto MHC class I of tumor cells was detected (Fig. 5A).

To evaluate the immunological response to intratumoral OVA peptide vaccine and $\alpha$ PD- $1 \mathrm{Ab}$, the spleens and tumors of mice treated with the same schedule were analyzed as described previously (Fig. 4A). Peptide-specific immune responses were detected in the spleens of mice treated with intratumoral OVA peptide injection using IFN- $\gamma$ ELISPOT assays (Fig. 5B). Mice that received the combination of intratumoral OVA peptide injection and $\alpha$ PD-1 Ab exhibited an increased number of OVA peptide-specific CTLs compared with those treated with control $\mathrm{Ab}$ on day $14(\mathrm{n}=10)$.

To obtain direct evidence that the combination of peptide vaccine and $\alpha \mathrm{PD}-1 \mathrm{Ab}$ led to the local accumulation of antigen-specific CTLs, an OVA tetramer assay was performed in mice. OVA-tetramer-positive CD8 lymphocytes could be detected within a tumor using flow cytometry on day 21 . Mice that received the combination of OVA peptide vaccine and $\alpha$ PD-1 Ab had a significantly increased number of OVA peptide-specific CTLs compared with those treated with control $\mathrm{Ab}$ (Fig. 5C and D; $\mathrm{n}=8$ ).

Inhibitory receptors on tumor-infiltrating T lymphocytes and the expression of chemokines. The expression of inhibitory receptors on peptide-specific CTLs at the tumor site was assessed to investigate the mechanism of CTL accumulation in the tumors of mice treated with the combination therapy of peptide vaccine and $\alpha$ PD- 1 Ab. RMA-bearing mice were treated with intratumoral OVA peptide injection combined with $\alpha \mathrm{PD}-1 \mathrm{Ab}$ or control $\mathrm{Ab}$, as described previously (Fig. 4A). The expression of PD-1, CTLA-4, and LAG-3 in OVA tetramer-positive CD8 lymphocytes within the tumor on day 21 was analyzed using flow cytometry. The expression of the inhibitory receptors PD- 1 and CTLA- 4 was decreased in OVA-tetramer positive CD8 lymphocytes in the $\alpha \mathrm{PD}-1 \mathrm{Ab}$ group compared with the control Ab group (Fig. 6A). However, $\alpha \mathrm{PD}-1 \mathrm{Ab}$ treatment did not decrease LAG-3 expression in OVA tetramer-positive CD8 lymphocytes.

The expression of chemokines within the tumor on day 21 was examined using quantitative real-time PCR. The expres- 
A
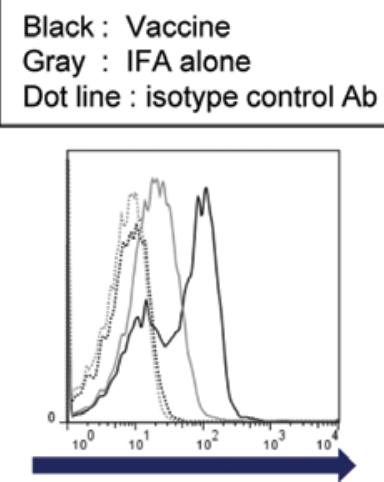

$\mathrm{K}^{\mathrm{b}}$ bound to $\mathrm{OVA}_{257-264}$ peptide
B

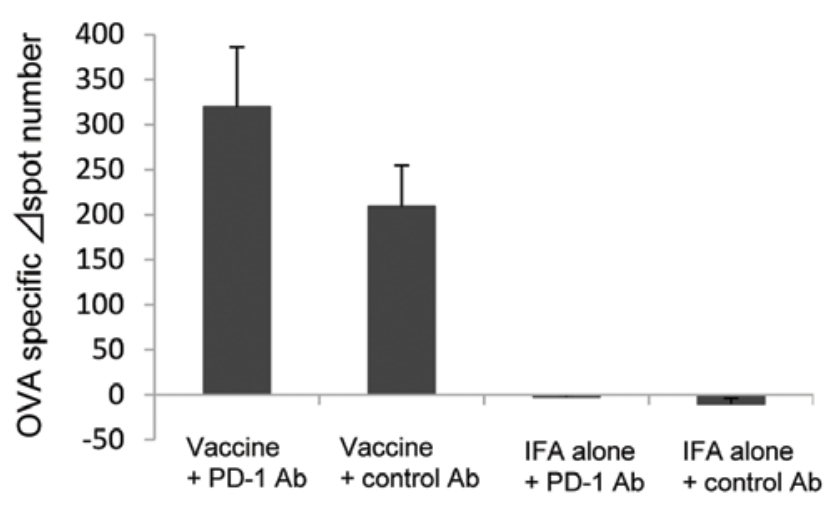

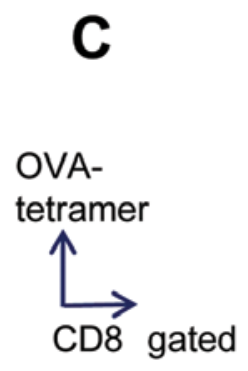

\section{Vaccine}

+ PD-1 Ab

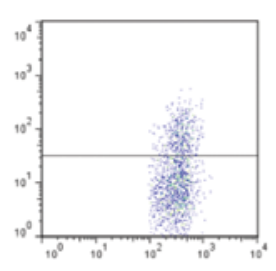

\section{Vaccine}

+ control $\mathrm{Ab}$

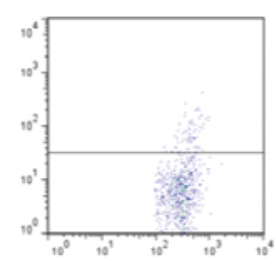

IFA alone

+ PD-1 Ab

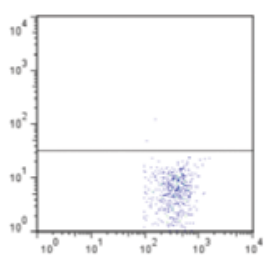

IFA alone + control $\mathrm{Ab}$

D

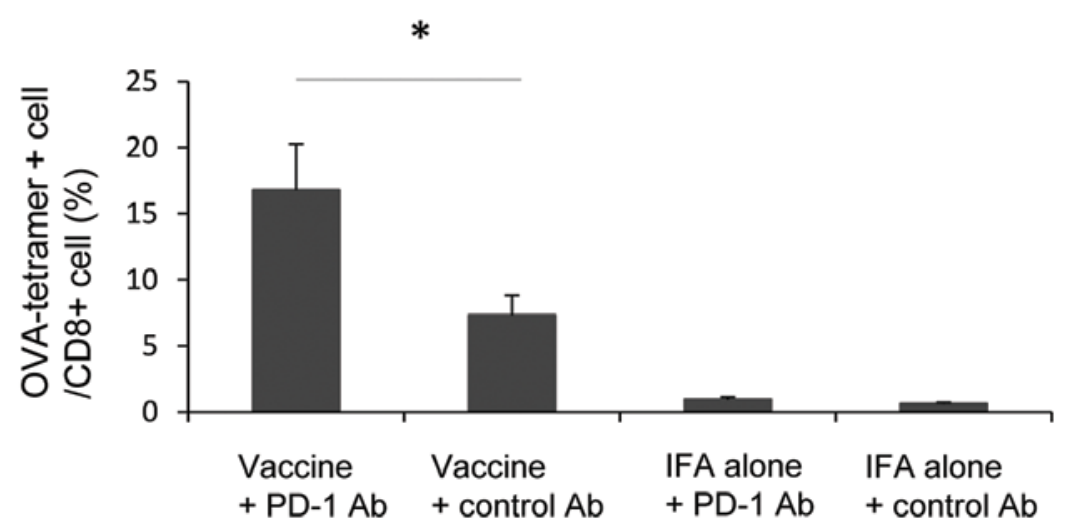

Figure 5. Blocking PD-1 enhanced the infiltration of vaccine-induced CTLs into the tumor. (A) Four days after the intratumoral injection with OVA $257-264$ peptide, isolated RMA tumor cells were stained with anti-mouse $\mathrm{H}-2 \mathrm{~K}^{\mathrm{b}}$ bound to $\mathrm{OVA}_{257-264}$ peptide or isotype control and analyzed using flow cytometry. Data are presented from a single representative sample $(\mathrm{n}=3)$. (B) RMA-bearing mice were treated with OVA peptide vaccine or IFA alone in combination with $\alpha \mathrm{PD}-1 \mathrm{Ab}$ or control Ab. Spleen cells from treated mice were analyzed using an ex vivo IFN- $\gamma$ ELISPOT assay on day 14. OVA-specific $\triangle$ spot number, spot number of $\mathrm{OVA}_{257-264}$ peptide pulsed BM-DC subtracted by non-pulsed BMDC. Data are presented as means \pm SEM (n=10). (C) Tumor-infiltrating T lymphocytes were analyzed using flow cytometry on day 21. Representative plots of OVA tetramer-positive, CD8-positive TILs in the tumors treated with the combination therapy of intratumoral OVA peptide injection and $\alpha \mathrm{PD}-1 \mathrm{Ab}$. (D) The percentages of OVA tetramer-positive cells in CD8-positive TILs are shown from three independent experiments using 2-3 mice per group. Data are presented as means $\pm \mathrm{SEM}$. $\mathrm{P}<0.05$, n=8 using Student's t-test.

sion of the chemokine CCL3 was elevated in mice treated with the combination of intratumoral OVA peptide injection and $\alpha \mathrm{PD}-1 \mathrm{Ab}$ (Fig. 6B). The expression of the chemokines CXCL10 and CXCL12 was unchanged.

\section{Discussion}

Many tumor antigens have been identified in HCC, and their potential clinical utility for the development of cancer-specific immunotherapy has been investigated (28-31). GPC3 is a promising target of antigen-specific immunotherapy because it is overexpressed specifically in human $\operatorname{HCC}(3,4)$. In addition, it promotes tumor growth by stimulating canonical Wnt signaling (32) or the Hippo pathway (33). A phase I clinical trial of a GPC3-derived peptide vaccine in patients with advanced HCC showed that it had the potential to improve overall survival, which was associated with vaccine-induced CTLs (8). However, the antitumor effects of the peptide-based tumor 
A

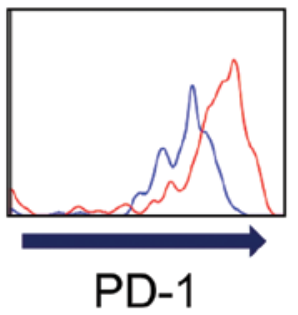

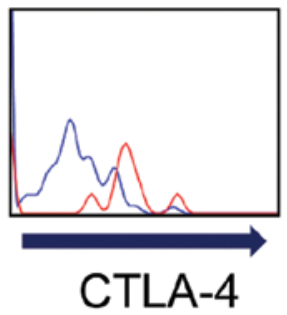

Blue: Vaccine + $\alpha \mathrm{PD}-1 \mathrm{Ab}$

Red: Vaccine + control $A b$

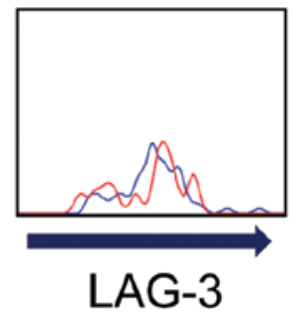

B
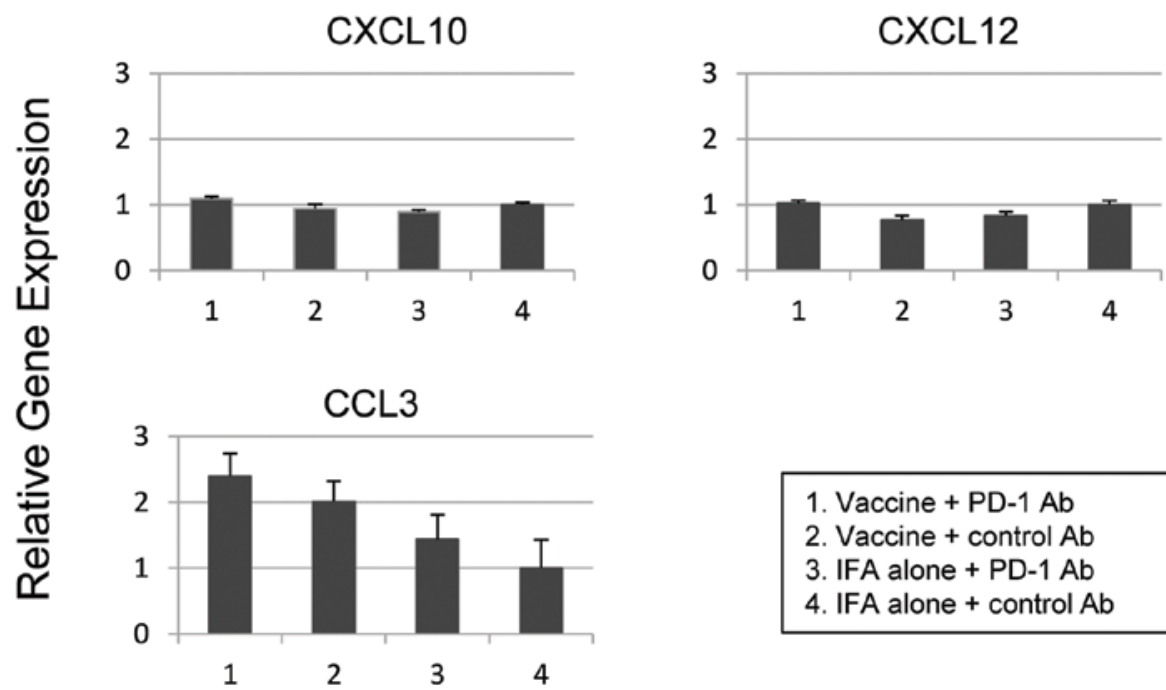

1. Vaccine + PD-1 Ab

2. Vaccine + control $A b$

3. IFA alone + PD-1 Ab

4. IFA alone + control $\mathrm{Ab}$

Figure 6. Changes in the expression of inhibitory receptors on tumor-infiltrating $\mathrm{T}$ lymphocytes and chemokines at the site of tumors treated using the combination therapy with peptide vaccine and $\alpha$ PD-1 Ab. RMA-bearing mice were treated with intratumoral OVA peptide injection combined with $\alpha$ PD- 1 Ab or control Ab. On day 21, mice were sacrificed and the tumors were isolated. (A) Histogram showing the expression of the inhibitory receptors PD-1, CTLA-4, and LAG-3 in OVA tetramer-positive CD8 lymphocytes in tumors from mice treated with intratumoral OVA peptide injection and $\alpha$ PD-1 Ab, as well as from mice treated with intratumoral OVA peptide injection and control Ab. Data are from a single representative sample (n=4-6). (B) The expression levels of chemokines in the tumor were analyzed using quantitative real-time PCR $(n=3)$. Relative expression levels in tumors treated with IFA alone and control Ab were calculated as the control. Data are presented as means \pm SEM. Two independent experiments were performed, which yielded similar results.

vaccine alone were not satisfactory in patients with advanced HCC (8,29-31). Several studies identified molecules associated with the tumor escape mechanism, such as PD-1/PD-L1, Fas/ FasL, and Decoy receptor 3, which might explain the poor immunogenicity and limitations of the antitumor effects of cancer vaccines alone in patients with advanced HCC $(16,17,34,35)$. Therefore, the present study examined whether blocking PD-1/PD-L enhanced the antitumor effects of peptide vaccines in HCC.

The inhibitory receptor PD-1, was upregulated in GPC3specific CTLs of HCC patients vaccinated using GPC3 peptide, consistent with previous reports of melanoma vaccine trials $(21,27)$. CTLs for some tumor antigens might not be detected directly ex vivo. The ex vivo analysis of antigen-specific CTLs from uncultured PBMCs could provide strong and novel immunological evidence in HCC vaccine trials. Fourcade et al reported that the upregulation of PD-1 and Tim-3 on CTLs was correlated with the expansion of melanoma-peptide vaccineinduced NY-ESO-1-specific CTLs (21). Further studies are necessary to understand the potential clinical efficacy of vaccine-induced CTLs.
In this experimental model, IFN- $\gamma$ induced PD-L1 expression in liver cancer cell lines. It was also demonstrated that blocking PD-1 increased the number of GPC3-specific CTL clones that degranulate against these liver cancer cell lines in vitro. These results suggest that blocking the interaction between PD-1 and PD-L1 enhanced the antitumor effects of CTL in liver cancer cells that evaded CTLs by expressing $\mathrm{PD}-\mathrm{L} 1$. In contrast, $\mathrm{Xu}$ et al reported that $\alpha \mathrm{PD}-\mathrm{L} 1$ or $\alpha C T L A-4$ Abs did not enhance cytokine secretion and the proliferation of peripheral GPC3-specific $\mathrm{CD}^{+} \mathrm{T}$-cell from HCC patients significantly (36). Differences in the effects of blocking PD-1 and PD-L1 might account for the differences between spontaneous GPC3-specific CTLs and vaccineinduced CTLs.

The combination of a peptide vaccine with $\alpha \mathrm{PD}-1 \mathrm{Ab}$ enhanced tumor suppression and antigen-specific $\mathrm{T}$ cell infiltration into the tumors of mouse models. The exact mechanisms by which CTLs accumulate into tumors by blocking PD-1 are unclear. A previous study in a mouse model of adoptive cell transfer demonstrated that blocking PD-1 increased the production of CXCL10 by bone marrow-derived myeloid cells, 
which enhanced the recruitment of CTLs in the tumor (25). We hypothesize that the $\alpha$ PD-1 Ab affected chemokine expression, which resulted in recruitment of vaccine-induced CTLs to the tumor. In the present study, the experimental model did not show a change in the expression of CXCL10. However, the expression of CCL3 was elevated by the combination treatment with vaccine and $\alpha$ PD- $1 \mathrm{Ab}$. Furthermore, blocking PD-1 decreased the expression of inhibitory receptors in peptidespecific CTLs at the tumor site. Recently, mouse models revealed that peptide/IFA vaccination increased the antigendriven expression of the inhibitory receptors PD-1, LAG-3, CTLA-4, and Tim-3 in CTLs, suggesting partial exhaustion (37). PD-1 blockade might be a rational strategy that could be used to rescue CTLs in a state of exhaustion. Interestingly, $\alpha$ PD-1 Ab therapy did not decrease LAG-3 expression in TILs; however, CTLA-4 expression was decreased, suggesting the partial rescue of CTL from exhaustion. A previous study reported that dual treatment with $\alpha$ LAG-3 and $\alpha$ PD- $1 \mathrm{Ab}$ was effective in mice with established tumors (38) as well as during the in vitro expansion of human NY-ESO-1-specific CTLs (39). Furthermore, Sierro et al reported that blocking both PD-1 and PD-L1 might further enhance the antitumor effects of tumor vaccines in mouse models (40).

Based on the results of this clinical trial, the GPC3 peptide vaccine has fewer side effects due to its antigen specificity (8). Enhancing GPC3 peptide vaccine therapy is considered to be promising in terms of sustained tumor control in HCC patients. These data suggest that use of $\alpha$ PD -1 Ab could enhance the antitumor effects of a peptide vaccine, and provide the foundation for the clinical development of a combination therapy.

\section{Acknowledgements}

We thank Kayoko Shoda for technical assistance. We also thank Dr Shigehisa Kitano (National Cancer Center), Masashi Minami, Takao Yoshida and Hirotsugu Takano (Ono Pharmaceutical Co.) for scientific advice. Y.S. would like to thank the Foundation for Promotion of Cancer Research (Japan) for the Third-Term Comprehensive Control Research for Cancer for the award of a research resident fellowship. This study was supported in part by the National Cancer Center Research and Development Fund (25-A-7), as well as Research for Promotion of Cancer Control Programmes, Research on Applying Health Technology, and Third Term Comprehensive Control Research for Cancer from the Ministry of Health, Labor and Welfare, Japan and a research funding from Ono Pharmaceutical Co., Ltd. T.N. is a scientific advisor for Ono Pharmaceutical Co., Ltd.

\section{References}

1. Breous E and Thimme R: Potential of immunotherapy for hepatocellular carcinoma. J Hepatol 54: 830-834, 2011.

2. Greten TF, Manns MP and Korangy F: Immunotherapy of hepatocellular carcinoma. J Hepatol 45: 868-878, 2006.

3. Nakatsura T, Yoshitake Y, Senju S, et al: Glypican-3, overexpressed specifically in human hepatocellular carcinoma, is a novel tumor marker. Biochem Biophys Res Commun 306: 16-25, 2003.

4. Capurro M, Wanless IR, Sherman M, et al: Glypican-3: a novel serum and histochemical marker for hepatocellular carcinoma. Gastroenterology 125: 89-97, 2003.
5. Nakatsura T, Komori H, Kubo T, et al: Mouse homologue of a novel human oncofetal antigen, glypican-3, evokes T-cellmediated tumor rejection without autoimmune reactions in mice. Clin Cancer Res 10: 8630-8640, 2004.

6. Komori H, Nakatsura T, Senju S, et al: Identification of HLA-A2or HLA-A24-restricted CTL epitopes possibly useful for glypican-3-specific immunotherapy of hepatocellular carcinoma. Clin Cancer Res 12: 2689-2697, 2006.

7. Shirakawa H, Suzuki H, Shimomura M, et al: Glypican-3 expression is correlated with poor prognosis in hepatocellular carcinoma. Cancer Sci 100: 1403-1407, 2009.

8. Sawada Y, Yoshikawa T, Nobuoka D, et al: Phase I trial of a glypican-3-derived peptide vaccine for advanced hepatocellular carcinoma: immunologic evidence and potential for improving overall survival. Clin Cancer Res 18: 3686-3696, 2012.

9. Yoshikawa T, Nakatsugawa M, Suzuki S, et al: HLA-A2restricted glypican-3 peptide-specific CTL clones induced by peptide vaccine show high avidity and antigen-specific killing activity against tumor cells. Cancer Sci 102: 918-925, 2011.

10. Agata Y, Kawasaki A, Nishimura H, et al: Expression of the PD-1 antigen on the surface of stimulated mouse $\mathrm{T}$ and $\mathrm{B}$ lymphocytes. Int Immunol 8: 765-772, 1996.

11. Freeman GJ, Long AJ, Iwai Y, et al: Engagement of the PD-1 immunoinhibitory receptor by a novel B7 family member leads to negative regulation of lymphocyte activation. J Exp Med 192: 1027-1034, 2000.

12. Iwai Y, Ishida M, Tanaka Y, Okazaki T, Honjo T and Minato N: Invovement of PD-L1 on tumor cells in the escape from host immune system and tumor immunotherapy by PD-L1 blockade. Proc Natl Acad Sci USA 99: 12294-12297, 2002.

13. Iwai Y, Terawaki S and Honjo T: PD-1 blockade inhibits hematogenous spread of poorly immunogenic tumor cells by enhanced recruitment of effector T cells. Int Immunol 17: 133-144, 2005.

14. Topalian SL, Hodi FS, Brahmer JR, et al: Safety, activity, and immune correlates of anti-PD-1 antibody in cancer. N Engl J Med 366: 2443-2454, 2012.

15. Wolchok JD, Kluger H, Callahan MK, et al: Nivolumab plus ipilimumab in advanced melanoma. N Engl J Med 369: 122-133, 2013.

16. Shi F, Shi M, Zeng Z, et al: PD-1 and PD-L1 upregulation promotes CD8(+) T-cell apoptosis and postoperative recurrence in hepatocellular carcinoma patients. Int J Cancer 128: 887-896, 2011.

17. Gao Q, Wang XY, Qiu SJ, et al: Overexpression of PD-L1 significantly associates with tumor aggressiveness and postoperative recurrence in human hepatocellular carcinoma. Clin Cancer Res 15: 971-979, 2009.

18. McGray AJ, Bernard D, Hallett R, et al: Combined vaccination and immunostimulatory antibodies provides durable cure of murine melanoma and induces transcriptional changes associated with positive outcome in human melanoma patients. Oncoimmunology 1: 419-431, 2012.

19. Mkrtichyan M, Najjar YG, Raulfs EC, et al: Anti-PD-1 synergizes with cyclophosphamide to induce potent antitumor vaccine effects through novel mechanisms. Eur J Immunol 41: 2977-2986, 2011.

20. Duraiswamy J, Kaluza KM, Freeman GJ and Coukos G: Dual blockade of PD-1 and CTLA-4 combined with tumor vaccine efficacy restorres $\mathrm{T}$ cell rejection function in tumors. Cancer Res 73: 3591-3603, 2013.

21. Fourcade J, Sun Z, Pagliano O, et al: PD-1 and Tim-3 regulate the expansion of tumor antigen-specific $\mathrm{CD} 8^{+} \mathrm{T}$ cells induced by melanoma vaccines. Cancer Res 74: 1045-1055, 2014.

22. Nobuoka D, Yoshikawa T, Takahashi M, et al: Intratumoral peptide injection enhances tumor cell antigenicity recognized by cytotoxic $\mathrm{T}$ lymphocytes: a potential option for improvement in antigen-specific cancer immunotherapy. Cancer Immunol Immunother 62: 639-652, 2013.

23. Li B, VanRoey M, Wang C, Chen TH, Korman A and Jooss K: Anti-programmed death-1 synergizes with granulocyte macrophage colony-stimulating factor - secreting tumor cell immunotherapy providing therapeutic benefit to mice with established tumors. Clin Cancer Res 15: 1623-1634, 2009.

24. Iwama T, Horie K, Yoshikawa T, et al: Identification of an $\mathrm{H} 2-\mathrm{K}^{\mathrm{b}}$ or $\mathrm{H} 2-\mathrm{D}^{\mathrm{b}}$ restricted and glypican-3-derived cytotoxic T-lymphocyte epitope peptide. Int J Oncol 42: 831-838, 2013.

25. Peng W, Liu C, Xu C, et al: PD-1 blockade enhances T-cell migration to tumors by elevating IFN- $\gamma$ inducible chemokines. Cancer Res 72: 5209-5218, 2012. 
26. Tada Y, Yoshikawa T, Shimomura M, et al: Analysis of cytotoxic $\mathrm{T}$ lymphocytes from a patient with hepatocellular carcinoma who showed a clinical response to vaccination with a glypican-3-derived peptide. Int J Oncol 43: 1019-1026, 2013.

27. Wong RM, Scotland RR, Lau RL, et al: Programmed death-1 blockade enhances expansion and functional capacity of human melanoma antigen-specific CTLs. Int Immunol 19: 1223-1234, 2007.

28. Mizukoshi E, Nakamoto Y, Arai K, et al: Comparative analysis of various tumor-associated antigen-specific T-cell responses in patients with hepatocellular carcinoma. Hepatology 53: 1206-1216, 2011

29. Butterfield LH, Ribas A, Meng WS, et al: T-cell responses to HLA-A*0201 immunodominant peptides derived from alphafetoprotein in patients with hepatocellular cancer. Clin Cancer Res 9: 5902-5908, 2003.

30. Butterfield LH, Ribas A, Dissette VB, et al: A phase I/II trial testing immunization of hepatocellular carcinoma patients with dendritic cells pulsed with four alpha-fetoprotein peptides. Clin Cancer Res 12: 2817-2825, 2006.

31. Greten TF, Forner A, Korangy F, et al: A phase II open trial evaluating safety and efficacy of a telomerase peptide vaccination in patients with advanced hepatocellular carcinoma. BMC Cancer 10: 209, 2010.

32. Capurro MI, Xiang YY, Lobe C and Filmus J: Glypican-3 promotes the growth of hepatocellular carcinoma by stimulating canonical Wnt signaling. Cancer Res 65: 6245-6254, 2005.

33. Feng M, Gao W, Wang R, et al: Therapeutically targeting glypican-3 via a conformation-specific single-domain antibody in hepatocellular carcinoma. Proc Natl Acad Sci USA 110: E1083-E1091, 2013.
34. Nagao M, Nakajima Y, Hisanaga M, et al: The alteration of Fas receptor and ligand system in hepatocellular carcinomas: how do hepatoma cells escape from the host immune surveillance in vivo? Hepatology 30: 413-421, 1999.

35. Chen C, Zhang C, Zhuang G, et al: Decoy receptor 3 overexpression and immunologic tolerance in hepatocellular carcinoma (HCC) development. Cancer Invest 26: 965-974, 2008.

36. Xu Y, Li H, Gao RL, Adeyemo O, Itkin M and Kaplan DE: Expansion of interferon-gamma-producing multifunctional $\mathrm{CD}^{+} \mathrm{T}$-cells and dysfunctional $\mathrm{CD}^{+} \mathrm{T}$-cells by glypican- 3 peptide library in hepatocellular carcinoma patients. Clin Immunol 139: 302-313, 2011.

37. Hailemichael Y, Dai Z, Jaffarzad N, et al: Persistent antigen at vaccination sites induces tumor-specific CD8(+) T cell sequestration, dysfunction and deletion. Nat Med 19: 465-472, 2013.

38. Woo SR, Turnis ME, Goldberg MV, et al: Immune inhibitory molecules LAG-3 and PD-1 synergistically regulate T-cell function to promote tumoral immune escape. Cancer Res 72: 917-927, 2012.

39. Matsuzaki J, Gnjatic S, Mhawech-Fauceglia P, et al: Tumorinfiltrating NY-ESO-1-specific $\mathrm{CD}^{+} \mathrm{T}$ cells are negatively regulated by LAG-3 and PD-1 in human ovarian cancer. Proc Natl Acad Sci USA 107: 7875-7880, 2010.

40. Sierro SR, Donda A, Perret R, et al: Combination of lentivector immunization and low-dose chemotherapy or PD-1/PD-L1 blocking primes self-reactive $\mathrm{T}$ cells and induces antitumor immunity. Eur J Immunol 41: 2217-2228, 2011. 